\title{
Is antibiotic lock therapy effective for the implantable long- term catheter-related bloodstream infections in children?
}

\author{
Tuğçe Tural Kara ${ }^{1}$, Halil Özdemir ${ }^{1}$, Tuğba Erat ${ }^{1}$, Aysun Yahşi ${ }^{1}$, Ahmet Derya Aysev ${ }^{2}$, \\ Nurdan Taçyıldız ${ }^{3}$, Emel Ünal ${ }^{3}$, Talia İleri ${ }^{4}$, Elif İnce ${ }^{4}$, Şule Haskoloğlu ${ }^{5}$, Ergin Çiftçi ${ }^{1}$, \\ Erdal İnce ${ }^{1}$ \\ Departments of ${ }^{1}$ Pediatric Infectious Diseases, ${ }^{2}$ Microbiology Laboratory, ${ }^{3}$ Pediatric Oncology, ${ }^{4}$ Pediatric Hematology and \\ ${ }_{5}^{5}$ Pediatric Immunology-Allergy, Ankara University Faculty of Medicine, Ankara, Turkey. E-mail: tugcetural@hotmail.com \\ Received: 8th July 2018, Revised: 26th October 2018, 24th January 2019, 14th May 2019, \\ Accepted: 15th May 2019
}

SUMMARY: Tural Kara T, Özdemir H, Erat T, Yahşi A, Aysev AD, Taçyıldız $\mathrm{N}$, Ünal E, İleri T, İnce E, Haskoloğlu Ş, Çiftçi E, İnce E. Is antibiotic lock therapy effective for the implantable long-term catheter-related bloodstream infections in children? Turk J Pediatr 2019; 61: 895-904.

Catheter-related bloodstream infections (CRBSIs) are an important problem in pediatric patients with central venous catheters. This study aimed to determine the incidence of CRBSIs, responsible pathogens and outcomes of antibiotic lock treatment (ALT) in pediatric patients. Between January 2010 and November 2015 all hospitalized pediatric hematology, oncology and immunology patients diagnosed with CRBSIs were retrospectively analyzed.

Seventy-eight CRBSI episodes were detected in 60 pediatric patients. The incidence of CRBSIs was $4.20 / 1000$ catheter days. The most frequently detected pathogen was methicillin-resistant coagulase-negative Staphylococcus. Pseudomonas aeruginosa, Klebsiella spp., and Escherichia coli were other commonly isolated microorganisms. ALT was administered in 42 patients. The success rate of ALT was $81 \%(34 / 42)$. Catheter was removed without ALT in 36 episodes. Common reasons for catheter removal were sepsis and causative microorganisms which had high probability of biofilm formation.

CRBSIs are an important cause of morbidity and mortality in pediatric patients. ALT is safe and effective. It is possible to obtain satisfactory results when ALT is used with intravenous systemic antibiotics for CRBSIs, though in some cases catheter removal is necessary. ALT helps to prevent unnecessary catheter removal in pediatric patients.

Key words: Antibiotic lock therapy, catheter-related bloodstream infections, central venous catheter.

Bloodstream infections (BSIs) are the most important problem in children with hematologic, oncologic and immunologic disease. In recent years, central venous catheter (CVC) use has increased because of blood sampling for laboratory evaluation, total parenteral nutrition and administration of chemotherapeutic drugs, blood products and fluids. ${ }^{1}$
Nowadays, central line related infections have become the most important problem in children with CVC which lead to increased morbidity and mortality resulting in prolonged and high cost of hospitalization. ${ }^{2-4}$ Central line related infections are divided into three groups according to localization; exit-site infection, tunnel infection and catheter-related bloodstream infection (CRBSI). ${ }^{5}$ 
Coagulase-negative Staphylococci (CoNS), Staphylococcus aureus, Enterococcus spp., Klebsiella spp. and other gram-negative microorganisms are detected as responsible pathogens for most CRBSIs in children. ${ }^{6}$ Microorganisms may be transmitted through the skin during catheter insertion. In addition, the catheter may be infected by close neighbors or via hematological routes. ${ }^{7}$ External surface colonization may be responsible for CRBSIs within 14 days after catheter placement and intraluminal colonization may cause infection after 14 days. Underlying disease, type of catheter and catheter placement localization are important determinants for development of CRBSIs. ${ }^{8}$ Children who are administered intensive chemotherapy and have external or double lumen catheters have high risk for CRBSI. $^{9}$

Antibiotic lock therapy (ALT) is a treatment method which involves high concentration of antibiotics inserted into the catheter lumens for sterilization of CRBSI. ${ }^{3}$ Microorganisms may create a pathogenic biofilm by reaching the intraluminal space and therefore they may cause CRBSI. Required antibiotic concentration should be 100-1000 times more than minimum inhibitory concentration to kill the responsible microorganisms in the catheter lumen. ${ }^{10}$ Intravenous antibiotic treatment is sometimes not sufficient to eradicate microorganisms and results in catheter removal to eliminate CRBSI. However, an attempt is made to reduce unnecessary catheter removal with ALT because of cost and re-catheterization complications. ${ }^{2,11}$

Herein we aimed to determine the incidence of CRBSIs and responsible pathogens, treatment modalities, outcomes of ALT, treatment failure, relapse, complications and mortality in pediatric immunocompromised patients.

\section{Material and Methods}

\section{Patients}

Hospitalized children who were diagnosed with CRBSI in the Pediatric Hematology, Oncology and Immunology Departments between January 2010 and November 2015 were retrospectively analyzed. Patients' demographic and clinical features, history of CVC, type of catheter, date of CVC implantation and removal, absolute neutrophil count, C-reactive protein level (CRP), isolated microorganism, antibiotic susceptibility, selected intravenous antibiotics, rate and duration of ALT, treatment failure, recurrence, catheter removal, complications and mortality were collected from the Hospital Discharge Data Collection System.

\section{Patient follow-up and treatment}

In our hospital, at least $2 \mathrm{~mL}$ blood samples were taken from peripheral vein and both catheter lumen for cultivation in patients with CVC who had fever (body temperature $>37.5^{\circ} \mathrm{C}$ at least 2 hours or $>38{ }^{\circ} \mathrm{C}$ one time $)^{12}$ without focus or unstable vital signs (tachycardia, tachypnea, hypotension, hypothermia, apnea or bradycardia). In addition, samples were taken from potential infected areas to identify the focus of infection. All blood cultures were taken before initiating of empiric antimicrobial therapy.

Systemic empirical antibiotic treatment was performed according to Infectious Disease Society of America (IDSA) guidelines (antipseudomonal broad-spectrum betalactamase antibiotics if absolute neutrophil count (ANC) $<500 / \mathrm{mm}^{3}$ and third generation cephalosporin if ANC $\left.>500 / \mathrm{mm}^{3}\right) .{ }^{5}$ Treatment was rearranged after identifying the causative microorganism. Aminoglycosides were used in high risk patients with malignancy. Glycopeptide antibiotics were not routinely used except for mucositis, pneumonia, soft tissue infections, and methicillin-resistant $S$. aureus colonization.

Catheter removal was performed under following conditions: S. aureus, Pseudomonas spp., fungus and mycobacteria related catheter infections, non-tunneled catheter infections, all tunnel infections, complicated catheter infections and in septic patients. If catheter removal was not required, ALT was used with systemic intravenous antibiotics in patients documented with CRBSI which includes concentrated antibiotic solution (vancomycin $5 \mathrm{mg} / \mathrm{mL}$, gentamicin $1 \mathrm{mg} / \mathrm{mL}$, amikacin 2 $\mathrm{mg} / \mathrm{mL}$, ciprofloxacin $0.2 \mathrm{mg} / \mathrm{mL}$, ampicillin $10 \mathrm{mg} / \mathrm{mL}$, ceftazidime $0.5 \mathrm{mg} / \mathrm{mL}$, cefazolin $5 \mathrm{mg} / \mathrm{mL}$ ), sterile normal saline and heparin 
(100 U/mL)..$^{5,13}$ Only one antibiotic was chosen in accordance with antimicrobial susceptibility. After the antibiotic and heparin to be given for ALT were prepared in the appropriate concentrations as indicated above, the remaining volume was completed with sterile normal saline to make the solution to be $3 \mathrm{~mL}$. This mixture was introduced into the catheter lumen (usually $3 \mathrm{~mL}$ ) and then the catheter was locked. Antibiotic lock solution was replaced every 24 hours. The duration of ALT varied according to the causative microorganisms; ALT treatment for Enteroccoccus spp. was 7-14 days, CoNS was 10-14 days, and gram negative bacilli was 10-14 days. ${ }^{5}$ Control catheter and peripheral blood cultures were performed after $72 \mathrm{~h}$ of treatment. Patients were followed up according to clinical signs. If fever was persistent and sterile blood culture could not be achieved, catheter was removed and catheter tip culture was performed. After diagnosis of CRBSI, all patients were followed for at least 6 months.

\section{Microbiology}

All blood samples were cultured with BACTEC 9240 system (Becton Dickinson, Sparks, MD, USA). Antimicrobial susceptibility of microorganisms was detected with Phoenix 100 ID/AST system (Becton Dickinson, Phoenix 100, MD, USA). Central venous catheter tip culture was performed by semiquantitative roll plate culture.

\section{Definitions}

Diagnosis of CRBSI was based on the IDSA Clinical and Practice Guidelines for the Diagnosis and Management of Intravascular Catheter-related Infections: 2009 updated version which contains the following criteria: detecting the same microorganism from catheter and peripheral blood culture, and microorganism reproduction time is detected at least two hours earlier in catheter blood culture than peripheral blood culture. ${ }^{5}$ If nonpathogenic microorganisms of normal skin flora (CoNS, Streptococcus viridans, Propionibacterium spp., Bacillus spp.) are isolated from at least two separate blood cultures, it is diagnosed as CRBSI. The clinical signs of infection were detected at the same time when the blood culture sample was taken.
The frequency of CRBSI was calculated as the number of episodes/1000 catheter days. Treatment failure was defined as continued growth of the same microorganism in blood culture within 72 hours of ALT. If the same pathogen was isolated from the blood culture within 3 months, it was accepted as relapse. ${ }^{13}$ Thirty days mortality (death occurring within 30 days of a defined diagnosis of a bloodstream infection) was used to identify deaths.

\section{Statistical analysis}

Results were analyzed by using the SPSS version 18.0 (Chicago, IL, USA). Chi-square test or Fisher's exact test were performed for categorical variables. Differences between continuous variables were identified with t test or Mann-Whitney $U$ test. A value of $p<0.05$ was considered statistically significant.

Approval for the study was obtained from the Ethics Committee of Ankara University Faculty of Medicine (2016/04).

\section{Results}

During study period, positive catheter blood cultures were detected in 88 episodes. Although catheter blood cultures were positive, peripheral blood cultures were negative in 10 of them. In addition, these patients did not have any clinical symptoms. Therefore, the catheter was considered colonized in these patients with positive cultures. Eventually 78 CRBSIs were detected in 60 pediatric hematology, oncology and immunology patients. Demographic and clinical characteristics of patients are summarized in Table I. The median age of patients was 86 (range, 3-240) months. Male/ female ratio was $1.9 / 1$. Types of catheters were as follows: $75.6 \%$ Hickman catheters and $24.4 \%$ totally implantable ports. The incidence of CRBSIs was 4.20/1000 catheter days. The incidence of CRBSIs was found to be similar for Hickman catheters and totally implantable ports (4.13/1000, 4.44/1000 catheter days). The incidence of CRBSIs between groups were as follows: immune deficiency 4.80/1000 catheter days, Acute Myeloblastic Leukemia (AML) 4.15/1000 catheter days, aplastic anemia 3.97/1000 catheter days, Acute Lymphoblastic Leukemia (ALL) 3.93/1000 catheter days, solid organ tumors 3.55/1000 
catheter days, lymphoma 3.21/1000 catheter days. The incidences of CRBSIs are given in Figure 1 between 2010 and 2015.
Neutropenia was observed in 56 (71.8\%) episodes and the median duration of neutropenia was 8 (range, 0-68) days. The

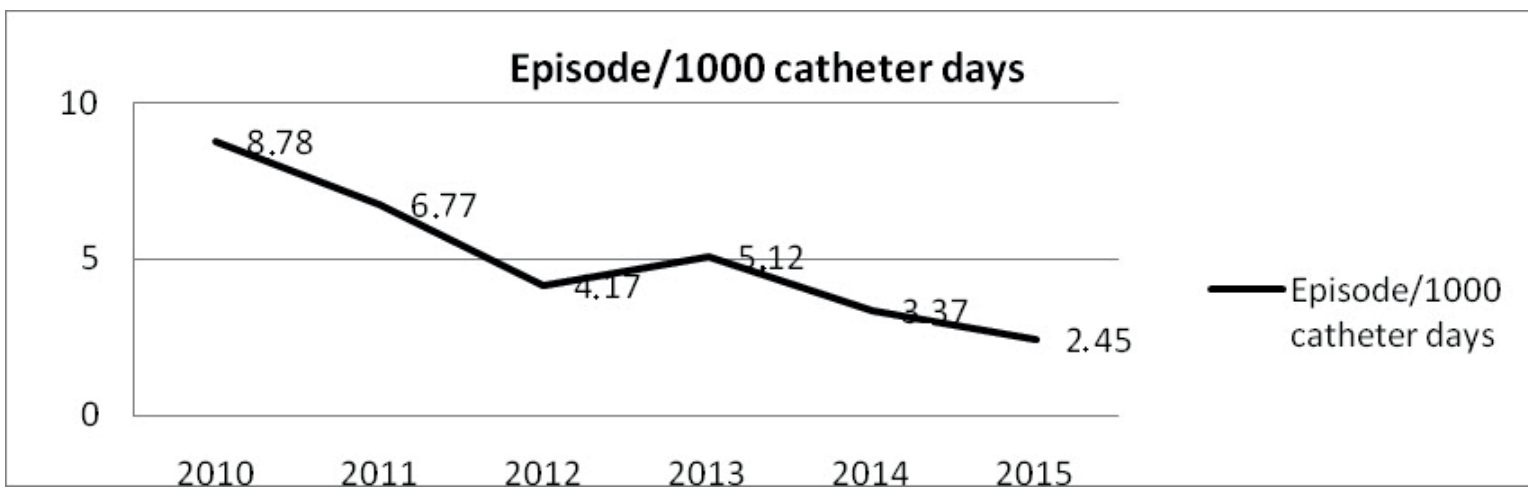

Fig. 1. The incidence of catheter-related bloodstream infections between 2010 and 2015.

Table I. Demographic and Clinical Characteristics of Patients with Catheter Related Bloodstream Infections.

\begin{tabular}{|c|c|c|c|}
\hline Characteristic & $\mathrm{n}$ & Patients underlying disease & $\mathrm{n}(\%)$ \\
\hline Total number of patients & 60 & Acute lymphoblastic leukemia & $21(35)$ \\
\hline Age, median (range), months & $86(3-240)$ & Acute myeloid leukemia & $15(25)$ \\
\hline Gender & $39 \mathrm{M} / 21 \mathrm{~F}$ & Solid organ tumors & $10(16.6)$ \\
\hline Catheter type & & Neuroblastoma & 3 \\
\hline Hickman & $59(75.6 \%)$ & Ewing's sarcoma & 2 \\
\hline Port & $19(24.4 \%)$ & Osteosarcoma & 1 \\
\hline Incidence of CRBSI & $4.20 / 1000$ catheter days & Chondrosarcoma & 1 \\
\hline Hickman & $4.13 / 1000$ catheter days & Multiple myeloma & 1 \\
\hline Port & $4.44 / 1000$ catheter days & Retinoblastoma & 1 \\
\hline CRP levels, mean $\pm \mathrm{SD}, \mathrm{mg} / \mathrm{L}$ & $84.2 \pm 78.0$ & Synovial sarcoma & 1 \\
\hline WBC count, mean $\pm \mathrm{SD}$, cell $/ \mathrm{mm}^{3}$ & $2297.4 \pm 3516.2$ & Immune deficiency & $6(10)$ \\
\hline ANC count, mean $\pm \mathrm{SD}$, cell $/ \mathrm{mm}^{3}$ & $1376.9 \pm 2900.9$ & SCID & 3 \\
\hline $\begin{array}{l}\text { Neutropenia duration, mean } \pm \mathrm{SD} \text {, } \\
\text { day }\end{array}$ & $11.0 \pm 13.7$ & CVID & 2 \\
\hline CRBSI & 78 & LAD tip3 & 1 \\
\hline Antibiotic lock therapy & $42(53.8 \%)$ & Aplastic anemia & $2(3.3)$ \\
\hline Success rate of ALT & $34 / 42(81 \%)$ & Lymphoma & $2(3.3)$ \\
\hline Catheter removal & $44(56.4 \%)$ & $\begin{array}{l}\text { Hemophagocytic } \\
\text { lymphohistiocytosis }\end{array}$ & $1(1.7)$ \\
\hline Relapse & $1(1.3 \%)$ & Chronic myeloid leukemia & $1(1.7)$ \\
\hline \multirow[t]{2}{*}{ Mortality } & $3(3.8 \%)$ & MDS & $1(1.7)$ \\
\hline & & Thalassemia major & $1(1.7)$ \\
\hline
\end{tabular}

ALT: Antibiotic lock treatment; ANC: Absolute neutrophil count; CRBSI: Catheter-related bloodstream infection; CRP: C-reactive protein; CVID: Common variable immunodeficiency; LAD: Leukocyte adhesion deficiency; MDS: Myelodysplastic syndromes; SCID: Severe Combined Immune Deficiency; SD: Standard deviation; WBC: White blood cell 
Table II. The Causative Microorganisms and Their Treatment Response.

\begin{tabular}{lcccccc}
\hline & $\mathrm{n}(\%)$ & ALT & $\begin{array}{c}\text { Removal } \\
\text { catheter }\end{array}$ & $\begin{array}{c}\text { Successful ALT } \\
(\%)\end{array}$ & Relapse & Mortality \\
\hline MRCoNS & $15(18.5)$ & 15 & 2 & $13(86.7)$ & 0 & 0 \\
Pseudomonas spp. & $12(14.8)$ & 0 & 12 & $0(0)$ & 0 & 0 \\
Klebsiella spp. & $10(12.3)$ & 7 & 5 & $5(71.4)$ & 0 & 1 \\
E. coli & $8(9.9)$ & 5 & 4 & $4(80)$ & 0 & 0 \\
Candida spp. & $6(7.4)$ & 0 & 6 & $0(0)$ & 0 & 0 \\
Alpha-hemolytic Streptococcus & $5(6.2)$ & 5 & 0 & $5(100)$ & 0 & 0 \\
Acinetobacter spp. & $5(6.2)$ & 2 & 4 & $1(50)$ & 1 & 1 \\
Enterococcus spp. & $3(3.7)$ & 0 & 3 & $0(100)$ & 0 & 0 \\
A.xylosoxidans & $3(3.7)$ & 3 & 2 & $1(50)$ & 0 & 0 \\
Serratia marcescens & $3(3.7)$ & 0 & 3 & $0(0)$ & 0 & 1 \\
Enterobacter spp. & $2(2.6)$ & 2 & 1 & $1(50)$ & 0 & 0 \\
S. aureus & $2(2.6)$ & 1 & 1 & $1(100)$ & 0 & 0 \\
S. maltophilia & $1(1.2)$ & 1 & 0 & $1(100)$ & 0 & 0 \\
Streptococcus oralis & $1(1.2)$ & 1 & 0 & $1(100)$ & 0 & 0 \\
Orchobactrum anthropi & $1(1.2)$ & 1 & 0 & $1(100)$ & 0 & 0 \\
Kingella kingae & $1(1.2)$ & 0 & 1 & $0(0)$ & 0 & 0 \\
Leuconostoc & $1(1.2)$ & 0 & 1 & $0(0)$ & 0 & 0 \\
Campylobacter jejuni & $1(1.2)$ & 0 & 1 & $0(0)$ & 0 & 0 \\
Salmonella enteritis & $1(1.2)$ & 1 & 0 & $1(100)$ & 0 & 0 \\
\hline AlT: Antbioic lock rets & 1 & & 0 & \\
\end{tabular}

ALT: Antibiotic lock treatment; MRCoNS: Methicillin resistant coagulase negative staphylococci

most common underlying disease was ALL (35\%), followed by AML (25\%), solid organ tumors $(16.7 \%)$, immune deficiency $(10 \%)$, aplastic anemia (3.3\%), and lymphoma $(3.3 \%)$. Underlying diseases of the patients are summarized in Table I.

The most frequently detected pathogen was methicillin resistant coagulase negative staphylococci (MRCoNS) (18.5\%). Pseudomonas aeruginosa (14.8\%), Klebsiella spp. (12.3\%), and Escherichia coli (9.9\%) were other commonly isolated microorganisms. Candida spp. was detected in 6 patients (Candida kefyr $(\mathrm{n}=1)$, Candida parapsilosis $(\mathrm{n}=2)$, Candida albicans $(\mathrm{n}=2)$, Candida krusei $(\mathrm{n}=1))$. Polymicrobial infections were seen in $3.8 \%(3 / 78)$ of episodes. These were; $C$. krusei and E. coli, Acinebacter baumannii and Ochrobactrum anthropi, and Acinetobacter junii and Achromobacter xylosoxidans (Table II).
In our hospital between 2010 and 2015, gramnegative microorganisms were more common than gram-positive microorganisms; however, between 2011 and 2012 gram-positive microorganisms were dominant in CRBSIs. In this period the incidence of gram-negative microorganisms decreased, but it increased again until 2016. Between 2010 and 2015 the distribution of isolated microorganisms is given in Figure 2.

Treatment modalities were decided according to causative microorganism, patients' clinical status and type of catheter. When $S$. aureus, Pseudomonas spp. and any fungus were detected as causative for CRBSI, the catheter was removed immediately. Additionally, catheter removal was performed for all tunnel infections, complicated catheter infections and in septic patients. 


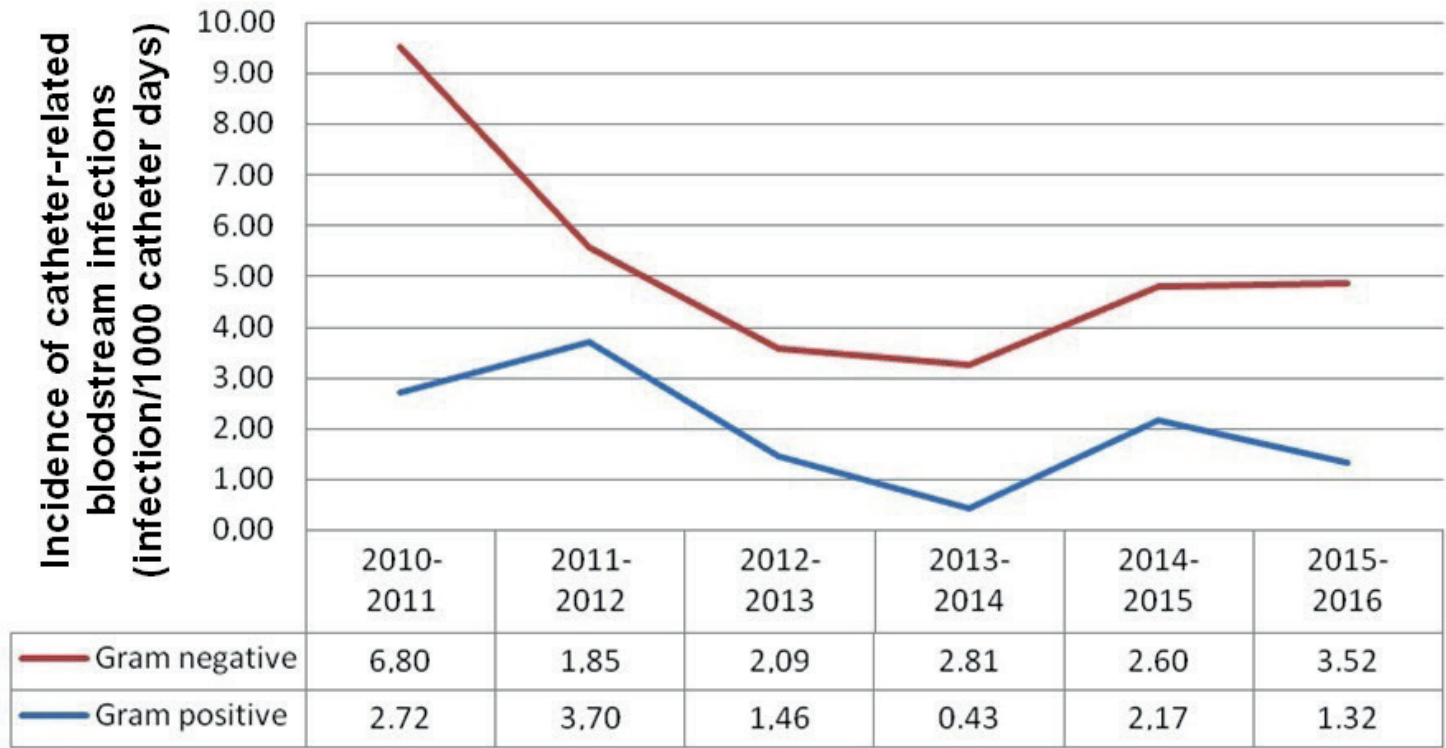

Fig. 2. According to year, causative microorganisms (gram-positive coccus and gram-negative bacilli) of catheter-related bloodstream infections (CRBSIs) in pediatric patients.

Standard intravenous antibiotic treatment was used in all CRBSI episodes. ALT was administered in addition to systemic intravenous antibiotic treatment in 42 patients. The catheter was removed in 8 of them who had treatment failure. Success rate of ALT was $81.0 \%$ (34/42). The catheter was removed without ALT treatment in 36 episodes. Reasons for catheter removal were as follows: microorganisms with high probability of biofilm formation (19/36), sepsis (15/36), multidrug resistant microorganisms (1/36), tunnel infection $(1 / 36)$ and thrombophlebitis $(1 / 36)$. S. aureus was detected in a patient with thrombophlebitis. This patient was included in both groups with thrombophlebitis and microorganisms with high probability biofilm formation.

Relapse was observed in 1 (1.3\%) episode. This patient had a Hickman catheter and his underlying disease was ALL. Acinetobacter spp. was detected in the relapse episode.

The rate of mortality was $3.8 \%(3 / 78)$ in all patients. Underlying diseases of these patients were as follows: AML $(n=1)$, neuroblastoma $(n=1)$, and congenital immune deficiency $(\mathrm{n}=1)$. Two patients received ALT and the rate of ALT-related mortality was $4.8 \%(2 / 42)$.

\section{Discussion}

Catheter-related bloodstream infections are an important problem in immunocompromised patients with CVC, due to prolonged hospitalization, increased morbidity, mortality and medical costs. Catheter removal is needed in some cases in spite of both systemic intravenous antibiotic treatment and ALT. In the literature, there is limited data about the success rate of ALT in pediatric patients. In this study, we identified the incidence and causative microorganisms of CRBSIs and effectiveness of ALT in pediatric hematology, oncology and immunology patients.

S. epidermidis and other CoNS were detected as the most commonly responsible microorganisms for CRBSIs. ${ }^{2,3,13}$ In our study MRCoNS, Pseudomonas spp., Klebsiella spp., and E. coli were most commonly isolated pathogens. We found that $33.3 \%$ grampositive microorganisms, $59.3 \%$ gramnegative microorganisms and $7.4 \%$ fungus were causative for CRBSIs. In the study by Celebi et al. ${ }^{14}$ of 31 pediatric hematology and oncology patients, $50 \%$ gram positive cocci, $31.6 \%$ gram negative bacilli and $4.5 \%$ fungi were detected in 44 CRBSIs and the rate of CRBSIs was 7.4/1000 catheter days. They suggested that CoNS was the most common 
causative pathogen because the main source of CRBSIs was contamination through the skin during catheter manipulations. Berrueco et al.'s ${ }^{15}$ study supported this hypothesis; they identified 25 CRBSIs in 73 children with acute leukemia and Staphylococcus spp. was the most commonly isolated pathogen. The rate of CRBSIs was reported as 6.7/1000 catheter days in 2007. After an educational program about hand hygiene this rate decreased to $1.5 / 1000$ catheter days. It was considered that educational programs and introduction of chlorhexidine $2 \%$ may help to achieve a low infection rate. Tsai et al. ${ }^{13}$ found the same result as us with Enterobacteriaceae $(40.2 \%)$ the predominant pathogen, followed by CoNS $(20.7 \%)$. They determined that using chlorhexidine reduced the incidence of CoNS-associated CRBSIs. Kao et al. ${ }^{16}$ detected skin cleansing with chlorhexidine helped to decrease gram-positive bacteria associated CRBSIs in adult patients with solid cancers.

In our hospital between 2010 and 2015, the incidence of CRBSIs was $4.20 / 1000$ catheter days and success of ALT was $81 \%$. In the literature, there are limited data on the efficiency of ALT in children. Wolf et al. ${ }^{2}$ compared 38 episodes of CRBSIs treated with ALT to 73 episodes treated with only standard antibiotic therapy in pediatric oncology patients. They found the success rate of ALT to be $50 \%$. Treatment failure was not significantly different between groups. However, timing of catheter removal was distinctive in these groups. Although immediate catheter removal was more often detected in systemic antibiotic therapy group, delayed removal and relapse of infections were more common in the ALT group. With all these results; they suggested that ALT was not more effective than systemic antibiotic therapy. We think ALT was not found to be effective because it was given without systemic intravenous antibiotic treatment. ALT alone was not sufficient to eliminate the infection.

In a study by Rijnders et al. ${ }^{17}$, adult and pediatric patients with CRBSIs who were hospitalized in hematology, oncology, gastroenterology and hemodialysis units were analyzed. In addition to systemic antibiotic therapy, 21 patients with ALT which contained vancomycin for gram-positive or ceftazidime for gram-negative bacteria were compared to 23 patients with placebo lock therapy. They identified using ALT may reduce treatment failure of CRBSI from $57 \%$ to $33 \%$. Relapse was detected in $9 / 23$ in placebo group and $3 / 21$ in ALT group. However, treatment failure and relapse were not significantly different between groups. This study showed that ALT does not provide benefit compared to systemic antibiotic therapy. They thought that even though the incidence of CRBSI was decreased, it was not statistically significant because of the following reasons: (i) the study was stopped early; because of this there were not enough patients to obtain sufficient statistical power, and (ii) ALT was thought to prevent relapse with the same strain but cure of the first episode of CRBSI did not increase in two weeks. Their study population included adult and pediatric patients with hematological, oncological, gastroenterological and kidney disease. Therefore; the efficiency of ALT may not be fully reflected in pediatric hematology and oncology patients.

In the literature, some studies have suggested that ALT is safe and helps to treat infected catheters with effective antibiotic concentration which is $100-5000$ times more than the minimum inhibitory concentration for causative pathogens. ${ }^{11,18}$ O'Horo et al. ${ }^{19}$ analyzed 8 studies which included adult and pediatric patients with CRBSIs. They found that both systemic antibiotic treatment and ALT obtained better results than only systemic antibiotic treatment. The rate of catheter removal was $10 \%$ in patients with ALT; this rate was $33 \%$ in patients with only systemic therapy. Rate of relapse was significantly lower in patients in the ALT group than in the nonALT group (20\% versus $30 \%$ ).

In Mermel et al.'s ${ }^{20}$ study children and adults with different underlying diseases had 681 CRBSIs episodes. They identified catheter salvage without recurrence was higher in the ALT group (82.6\%) than among patients who received systemic antibiotics alone $(66.5 \%)$. However, the generalization of this study is not true for pediatric patients with malignancy because of the heterogeneous study population. Tsai et al. ${ }^{13}$ examined 66 pediatric patients 
with hematology and oncology diseases. The incidence of CRBSI was 4.49/1000 catheter days and the success rate of ALT was $71.6 \%$. They suggested ALT should be used for routine treatment of CRBSI in pediatric hematologyoncology patients. The response to ALT was found to be low only in patients with older age, ALL, candidemia and elevated CRP levels.

Del Pozo et al. ${ }^{21}$ analyzed the effectiveness of ALT in 18 adult patients with port-related BSIs. The clinical and microbiologic success of ALT was $88 \%$. They found that if ALT was combined with systemic therapy, it was safe and effective for treatment. In Moreno et al.'s ${ }^{22}$ study linezolid lock therapy was used in 7 children with acute leukemia. Although intravenous teicoplanin or vancomycin treatment was not effective in 6 patients, linezolid ALT was successful against CoNS. They suggested that ALT and systemic therapy with linezolid may decreased the catheter removal in pediatric patients.

Fernandez-Hidalgo et al. ${ }^{23}$ analyzed 98 adult patients with 115 episodes of CRBSIs. They reported if ALT was combined with systemic antibiotic treatment, it was $82 \%$ effective for CRBSIs especially those caused by gramnegative pathogens and CoNS. In addition, $S$. aureus was found to be a major cause of high treatment failure.

Fortún et al. $^{24}$ reported 48 CRBSIs in 105 adult patients which were treated with ALT and systemic antibiotics (19/48) and only with systemic antibiotics (29/48). They found the success rate of ALT was $84 \%$ and $65 \%$ respectively and it showed that ALT was more effective for the management of CRBSIs. In Funalleras et al. ${ }^{25}$ study of 46 adult patients with CRBSIs due to gram-negative bacilli, patients were treated with ALT and systemic antibiotic treatment. They found the cure rate was $95 \%$ in 37 patients who completed followup. Therefore, they suggested that ALT and systemic antibiotic treatment combination were safe and effective for treatment of gram negative bacilli related CRBSIs.

In our study, the rate of catheter removal was $56.4 \%$ (44/78). In Adler et al.'s ${ }^{3}$ study 112 pediatric hematology and oncology patients were analyzed. The rate of CRBSI was $2.26 / 1000$ catheter days. They found catheter removal was $46 \%$ in patients during 207 CRBSI episodes. The catheter removal was for the following reasons: septic shock (13/96), Candida spp. infection (10/96), Mycobacterium spp. infection (7/96), persistent bacteremia (48/96), local infection (18/96) and reinfection (4/96). Although catheter removal was found at a higher rate, persistent bacteremia constituted only $50 \%$ of the reasons. In our study the catheter was removed in 19 patients because of the causative agent (Candida spp., $S$. aureus, Pseudomonas spp.). When the causative microorganisms were excluded from the analysis, this rate declined to $42.4 \%(25 / 59)$.

ALT may cause some adverse reactions such as antimicrobial resistance, secondary fungal infections, catheter occlusion and toxicity of applied antibiotics, anticoagulants and alcohol. ${ }^{21,26}$ By flushing the catheter, the patient may be exposed to unnecessary antibiotics and anticoagulants which may result in antimicrobial resistance and bleeding. ${ }^{26,27}$ Although no side effects have been reported for short-term use, sensorineural hearing loss has been reported in patients undergoing long-term amikacin lock treatment. ${ }^{28}$ In our study we did not see any ALT related side effects. In addition to antibiotic therapy; using heparin helped to prevent local side effects, such as catheter dysfunction and catheter obstruction. The lock solution contained 100 $\mathrm{U} / \mathrm{mL}$ heparin. Therefore, we believe that ALT is an effective and safe treatment. There are limited studies which report the outcomes of ALT in childhood. This is the first study in our country which includes the effectiveness of ALT. It is important to demonstrate that ALT may present good results by using indications which are defined in the IDSA guidelines.

In conclusion, CRBSI is an important cause of morbidity and mortality in pediatric hematology, oncology and immunology patients with CVC. MRCoNS was the most common causative pathogen. ALT is safe and helps to prevent unnecessary catheter removal in pediatric hematology, oncology and immunology patients when administered together with systemic treatment. 


\section{REFERENCES}

1. Chesshyre E, Goff Z, Bowen A, Carapetis J. The prevention, diagnosis and management of central venous line infections in children. J Infect 2015; 71(Suppl 1): S59-S75.

2. Wolf J, Allison KJ, Tang L, Sun Y, Hayden RT, Flynn PM. No evidence of benefit from antibiotic lock therapy in pediatric oncology patients with central line-related bloodstream infection: results of a retrospective matched cohort study and review of the literature. Pediatr Blood Cancer 2014; 61: 1811-1815.

3. Adler A, Yaniv I, Solter E, et al. Catheter-associated bloodstream infections in pediatric hematologyoncology patients: factors associated with catheter removal and recurrence. J Pediatr Hematol Oncol 2006; 28: 23-28.

4. Goudie A, Dynan L, Brady PW, Rettiganti M. Attributable cost and length of stay for central lineassociated bloodstream infections. Pediatrics 2014; 133: e1525-e1532.

5. Mermel LA, Allon M, Bouza E, et al. Clinical practice guidelines for the diagnosis and management of intravascular catheter-related infection: 2009 Update by the Infectious Diseases Society of America. Clin Infect Dis 2009; 49: 1-45.

6. Sievert DM, Ricks P, Edwards JR, et al; National Healthcare Safety. Network (NHSN) Team and Participating NHSN Facilities. Antimicrobialresistant pathogens associated with healthcareassociated infections: summary of data reported to the National Healthcare Safety Network at the Centers for Disease Control and Prevention, 20092010. Infect Control Hosp Epidemiol 2013; 34: 1-14.

7. Flynn PM. Diagnosis and management of central venous catheter-related bloodstream infections in pediatric patients. Pediatr Infect Dis J 2009; 28: 1016 1017.

8. Cecinati V, Brescia L, Tagliaferri L, Giordano P, Esposito S. Catheter-related infections in pediatric patients with cancer. Eur J Clin Microbiol Infect Dis 2012; 31: 2869-2877.

9. Yacobovich J, Ben-Ami T, Abdalla T, et al. Patient and central venous catheter related risk factors for blood stream infections in children receiving chemotherapy. Pediatr Blood Cancer 2015; 62: 471-476.

10. Ceri H, Olson ME, Stremick C, Read RR, Morck D, Buret A. The Calgary Biofilm Device: new technology for rapid determination of antibiotic susceptibilities of bacterial biofilms. J Clin Microbiol 1999; 37: 17711776 .

11. De Sio L, Jenkner A, Milano GM, et al. Antibiotic lock with vancomycin and urokinase can successfully treat colonized central venous catheters in pediatric cancer patients. Pediatr Infect Dis J 2004; 23: 963-965.
12. Kar YD, Özdemir ZC, Bör Ö. Evaluation of febrile neutropenic attacks of pediatric hematology-oncology patients. Turk Pediatri Ars 2017; 52: 213-220.

13. Tsai HC, Huang LM, Chang LY, et al. Central venous catheter-associated bloodstream infections in pediatric hematology-oncology patients and effectiveness of antimicrobial lock therapy. J Microbiol Immunol Infect 2015; 48: 639-646.

14. Celebi S, Sezgin ME, Cakır D, et al. Catheterassociated bloodstream infections in pediatric hematology-oncology patients. Pediatr Hematol Oncol 2013; 30: 187-194.

15. Berrueco R, Rives $\mathrm{S}$, Català $\mathrm{A}$, et al. Prospective surveillance study of blood stream infections associated with central venous access devices (port-type) in children with acute leukemia: an intervention program. J Pediatr Hematol Oncol 2013; 35: e194-e199.

16. Kao HF, Chen IC, Hsu C, et al. Chlorhexidine for the prevention of bloodstream infection associated with totally implantable venous ports in patients with solid cancers. Support Care Cancer 2014; 22: 11891197.

17. Rijnders BJ, Van Wijngaerden E, Vandecasteele SJ, Stas M, Peetermans WE. Treatment of long-term intravascular catheter-related bacteraemia with antibiotic lock: randomized, placebo-controlled trial. J Antimicrob Chemother 2005; 55: 90-94.

18. Carratalà J. The antibiotic-lock technique for therapy of 'highly needed' infected catheters. Clin Microbiol Infect 2002; 8: 282-289.

19. O'Horo JC, Silva GL, Safdar N. Anti-infective locks for treatment of central line-associated bloodstream infection: a systematic review and meta-analysis. Am J Nephrol 2011; 34: 415-422.

20. Mermel LA, Farr BM, Sherertz RJ, et al; Infectious Diseases Society of America; American College of Critical Care Medicine; Society for Healthcare Epidemiology of America. Guidelines for the management of intravascular catheter-related infections. Infect Control Hosp Epidemiol 2001; 22: 222-242.

21. Del Pozo JL, Alonso M, Serrera A, Hernaez S, Aguinaga A, Leiva J. Effectiveness of the antibiotic lock therapy for the treatment of port-related enterococci, Gram-negative, or Gram-positive bacilli bloodstream infections. Diagn Microbiol Infect Dis 2009; 63: 208-212.

22. Moreno RB, Rives S, Justicia A, et al. Successful porta-cath salvage using linezolid in children with acute leukemia. Pediatr Blood Cancer 2013; 60: E103-E105.

23. Fernandez-Hidalgo N, Almirante B, Calleja R, et al. Antibiotic-lock therapy for long-term intravascular catheter-related bacteraemia: results of an open, noncomparative study. J Antimicrob Chemother 2006; 57: 1172-1180. 
24. Fortún J, Grill F, Martín-Dávila P, et al. Treatment of long-term intravascular catheter-related bacteraemia with antibiotic-lock therapy. J Antimicrob Chemother 2006; 58: 816-821

25. Funalleras G, Fernández-Hidalgo N, Borrego A, et al. Effectiveness of antibiotic-lock therapy for long-term catheter-related bacteremia due to Gram-negative bacilli: a prospective observational study. Clin Infect Dis 2011; 53: e129-e132.

26. Justo JA, Bookstaver PB. Antibiotic lock therapy: review of technique and logistical challenges. Infect Drug Resist 2014; 7: 343-363.
27. Yevzlin AS, Sanchez RJ, Hiatt JG, et al. Concentrated heparin lock is associated with major bleeding complications after tunneled hemodialysis catheter placement. Semin Dial 2007; 20: 351-354.

28. Saxena AK, Panhotra BR, Naguib M. Sudden irreversible sensory-neural hearing loss in a patient with diabetes receiving amikacin as an antibioticheparin lock. Pharmacotherapy 2002; 22: 105-108. 